\title{
Editorial
}

\section{Nonlinear Analysis: Optimization Methods, Convergence Theory, and Applications}

\author{
Gonglin Yuan, ${ }^{1}$ Gaohang Yu, ${ }^{2}$ Neculai Andrei, ${ }^{3}$ Yunhai Xiao, ${ }^{4}$ and Li Zhang \\ ${ }^{1}$ College of Mathematics and Information Science, Guangxi University, Nanning, Guangxi 530004, China \\ ${ }^{2}$ School of Mathematics and Computer Sciences, Gannan Normal University, Ganzhou 341000, China \\ ${ }^{3}$ Research Institute for Informatics (ICI), 8-10 Averescu Avenue, 71316 Bucharest 1, Romania \\ ${ }^{4}$ College of Mathematics and Information Science, Henan University, Kaifeng 475000, China \\ ${ }^{5}$ Department of Mathematics, Changsha University of Science and Technology, Changsha 410004, China
}

Correspondence should be addressed to Gonglin Yuan; glyuan@gxu.edu.cn

Received 1 March 2015; Accepted 1 March 2015

Copyright (C) 2015 Gonglin Yuan et al. This is an open access article distributed under the Creative Commons Attribution License, which permits unrestricted use, distribution, and reproduction in any medium, provided the original work is properly cited.

Nonlinear analysis has been used in many practical application fields, such as nonlinear fitting, economics, optimization, convergence, engineering, hydrodynamics, parameter estimating, function approximating, and elasticity. There are many achievements on nonlinear analysis that have been obtained by authors. However, there still exist lots of challenging problems, such as the large-scale problems, fast algorithm, and convergence, since the complex of the nonlinear object function on its variables cannot be obviously determined in many cases. So the research and application space of nonlinear analysis are broad.

The issue invites investigators to contribute original research articles as well as review articles that will help in understanding the important new developments in nonlinear analysis and its applications with a particular emphasis on the following potential topics. There exist many special topics including the nonlinear analysis: optimization, variation analysis, economical models, fixed point theory, numerical methods, convergence, nonlinear equations, semidefinite programming, polynomial optimization, tensor computation, image processing, and so forth.

The research papers are welcome with new ideas or good numerical experiments. (1) New methods for nonlinear analysis are encouraged, such as the new formulas on conjugate gradient methods, quasi-Newton methods, limited memory
quasi-Newton method, trust region methods, and SQP methods; convergence results of algorithms are established which is needed. (2) Numerical experiments should be done to improve the theory idea: for unconstrained optimization problems, the CUTEr problems should be tested $[1,2]$ in Table 1. For nonlinear equations problems, there are many problems [3-7] that are listed in Table 2.

We hope that readers of this special issue will find not only convergence results and updated reviews on the common nonlinear analysis, but also important open problems to be resolved such as new formulas in optimization methods, new algorithms for variation analysis and new models for economic problems. Moreover, large-scale problems in nonlinear equations, semidefinite programming, and image processing are tested to turn out the performance of the new methods.
Gonglin Yuan Gaohang Yu

Neculai Andrei

Yunhai Xiao Li Zhang 
TABLE 1

\begin{tabular}{l}
\hline Problems names \\
\hline ARGLINA, ARGLINB, ARGLINC, BDQRTIC, BROWNAL, BROYDN7D, BRYBND CHAINWOO, CHNROSNB, \\
COSINE, CRAGGLVY, CURLY10, CURLY20, DIXMAANA, DIXMAANB, DIXMAANC, DIXMAAND, \\
DIXMAANE, DIXMAANF, DIXMAANG, DIXMAANH DIXMAANI, DIXMAANJ, DIXMAANL, DIXON3DQ, \\
DQDRTIC, DQRTIC, EDENSCH EG2, ENGVAL1, ERRINROS, EXTROSNB, FLETCBV2, FLETCHCR, \\
FREUROTH GENHUMPS, GENROSE, INDEF, LIARWHD, MANCINO, MSQRTALS, MSQRTBLS \\
NONCVXU2, NONDIA, NONDQUAR, PENALTY1, PENALTY2, POWELLSG POWER, QUARTC, \\
SCHMVETT, SENSORS, SINQUAD, SPARSINE, SPARSQUR SPMSRTLS, SROSENBR, TESTQUAD, TOINTGSS, \\
TQUARTIC, TRIDIA VARDIM, VAREIGVL, and WOODS \\
\hline DECONVU, FMINSRF2, FMINSURF, MOREBV, TOINTGOR, and TOINTQOR
\end{tabular}

TABLE 2

Functions names

Optimization value

Exponential function 1, exponential function 2, trigonometric function, singular function, logarithmic function,

Broyden tridiagonal function, trigexp function, strictly convex function 1, linear function-full rank, penalty function,

variable dimensioned function, tridiagonal system, five-diagonal system, extended Freudenstein and Roth function,

discrete boundary value problem, Troesch problem, and so forth

\section{References}

[1] I. Bongartz, A. R. Conn, N. I. Gould, and P. L. Toint, "CUTE: constrained and unconstrained testing environment," $A C M$ Transactions on Mathematical Software, vol. 21, no. 1, pp. 123160, 1995.

[2] N. I. M. Gould, D. Orban, and P. L. Toint, "CUTEr and sifdec: a constrained and unconstrained testing environment, revisited," ACM Transactions on Mathematical Software, vol. 29, no. 4, pp. 373-394, 2003.

[3] M. A. Gomes-Ruggiero, J. M. Martínez, and A. C. Moretti, "Comparing algorithms for solving sparse nonlinear systems of equations," SIAM Journal on Scientific and Statistical Computing, vol. 13, no. 2, pp. 459-483, 1992.

[4] G. Y. Li, "Successive column correction algorithms for solving sparse nonlinear systems of equations," Mathematical Programming, vol. 43, no. 2, pp. 187-207, 1989.

[5] B. Yang and L. Gao, "An efficient implementation of Merrill's method for sparse or partially separable systems of nonlinear equations," SIAM Journal on Optimization, vol. 1, no. 2, pp. 206221, 1991.

[6] J. J. Moré, B. S. Garbow, and K. E. Hillstrom, "Testing unconstrained optimization software," ACM Transactions on Mathematical Software, vol. 7, no. 1, pp. 17-41, 1981.

[7] S. M. Roberts and J. S. Shipman, "On the closed form solution of Troesch's problem," Journal of Computational Physics, vol. 21, no. 3, pp. 291-304, 1976. 


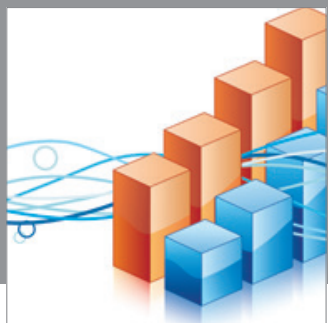

Advances in

Operations Research

mansans

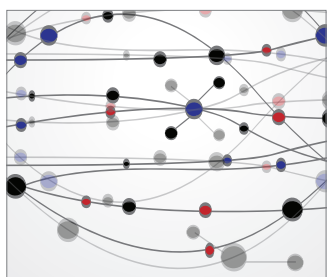

The Scientific World Journal
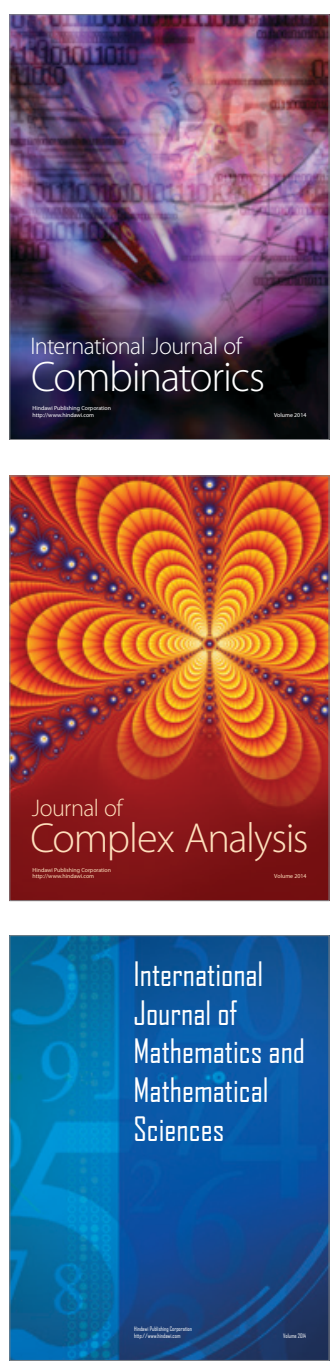
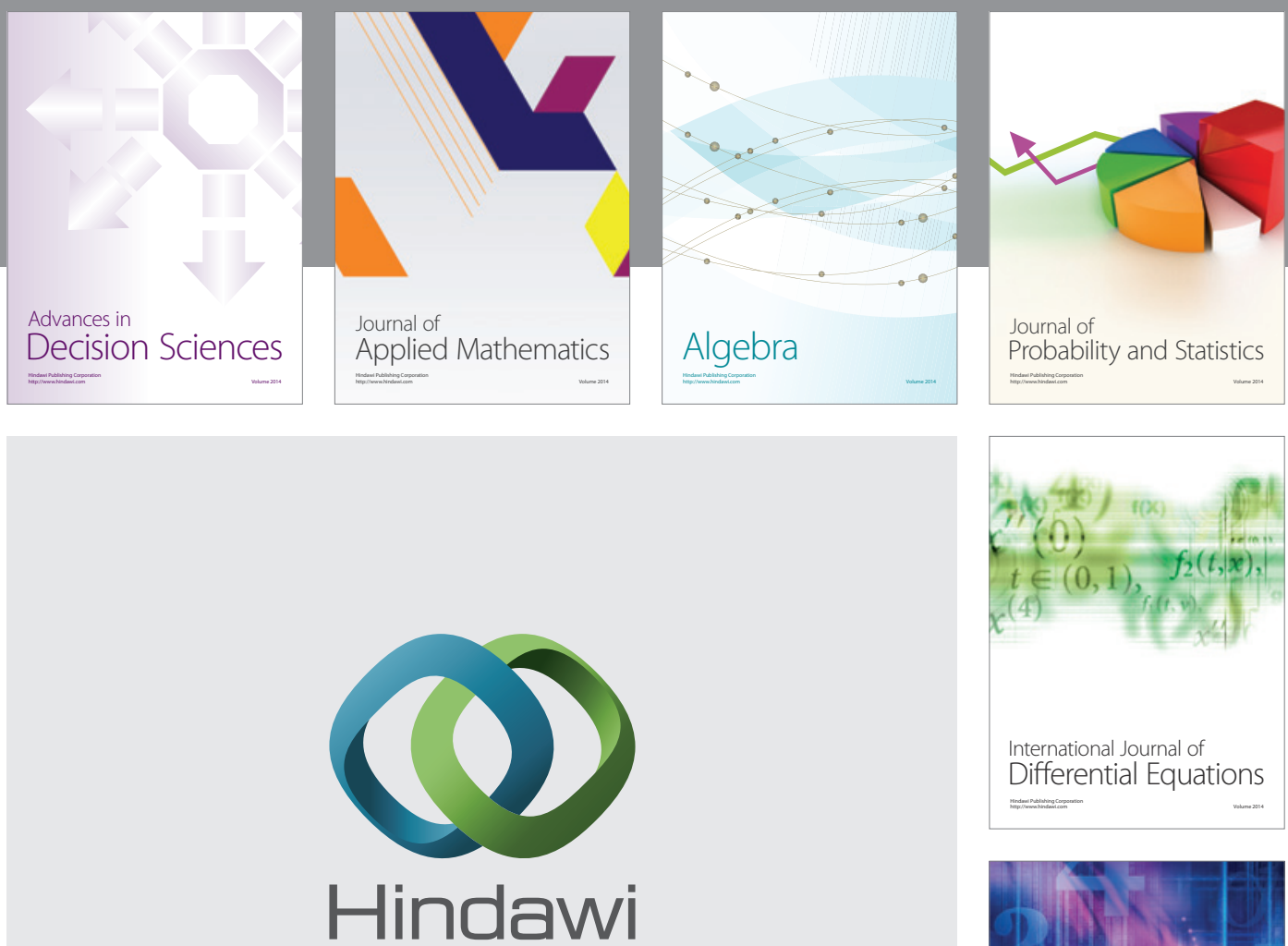

Submit your manuscripts at http://www.hindawi.com
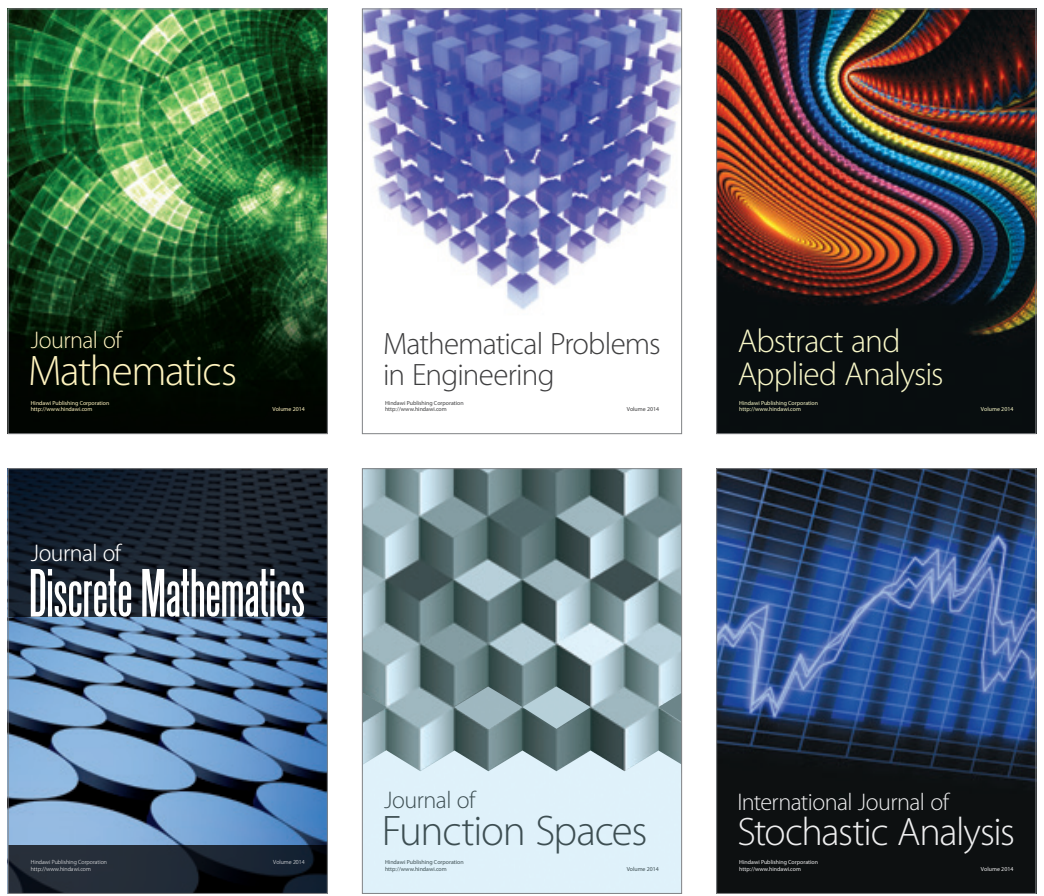

Journal of

Function Spaces

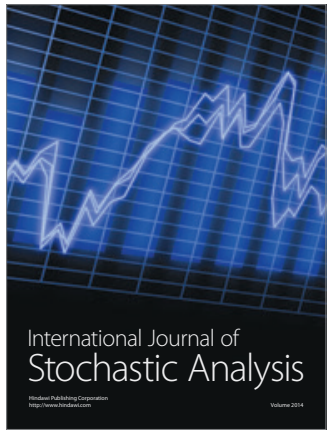

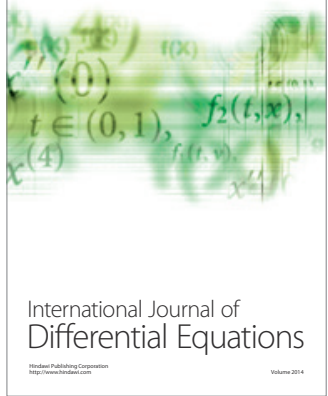
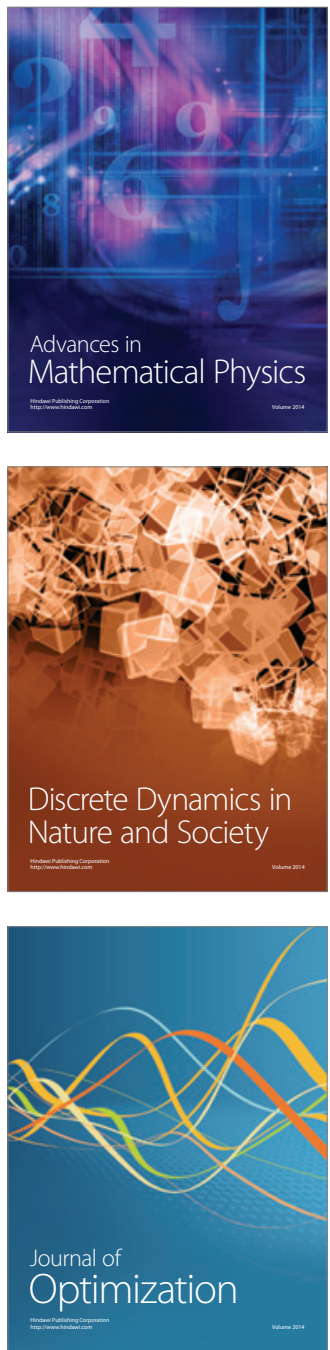